PROCEEDINGS OF THE

AMERICAN MATHEMATICAL SOCIETY

Volume 33, Number 2, June 1972

\title{
ON WEYL'S THEOREM AND ITS CONVERSE
}

\author{
MATTHEW C. Y. LEE ${ }^{1}$
}

\begin{abstract}
In this paper we study Weyl's theorem and von Neumann's converse of Weyl's theorem for the classes of all operators of the form $T_{f}^{-1} T_{a}$ and of the form $T_{q} T_{f}^{-1}$, where $T_{g}$ and $T_{f}$ are Toeplitz operators such that $T_{j}$ is invertible; and we can prove that Weyl's theorem holds for $T_{f}^{-1} T_{g}$ and for $T_{q} T_{f}^{-1}$.
\end{abstract}

Let $L(H)$ be the algebra of all bounded operators on an infinitedimensional complex Hilberi space $H$, and let $K(H)$ be the closed ideal of compact operators on $H$. We define the Weyl spectrum $\omega(A)$ to be $\cap \sigma(A+K)$, where $\sigma(A)$ denotes the spectrum of $A$ in $L(H)$ and the intersection is taken over all $K$ in $K(H)$. We say that Weyl's theorem holds for $A$ if $\sigma(A)-\omega(A)=\sigma_{00}(A)$, where $\sigma_{00}(A)$ denotes the set of all isolated eigenvalues of finite multiplicity of $A$. It is known that Weyl's theorem holds for any seminormal operator [1] and for any Toeplitz operator [4]. In 1935 J. von Neumann proved the following striking converse of Weyl's classical theorem [7, Satz II]: If $A$ and $B$ are selfadjoint operators in $L(H)$ such that $\omega(A)=\omega(B)$, then there exists a unitary operator $U$ in $L(H)$ such that $U A U^{*}-B$ is compact. Following S. K. Berberian [2] we say that $A$. $B \in L(H)$ are essentially equivalent if there exists a unitary operator $U$ in $L(H)$ such that $U_{A} U^{*}-B$ is compact. It is known that if $A$ and $B$ are normal operators such that $\omega(A)=\omega(B)$, then $A$ and $B$ are essentially equivalent (see e.g. [9]).

Let $L^{2}$ and $L^{\infty}$ denote the Lebesgue spaces of square-integrable and essentially bounded functions with respect to normalized Lebesgue measure on the unit circle in the complex plane. Let $H^{2}$ and $H^{\infty}$ denote the corresponding Hardy spaces. If $\varphi \in L^{\infty}$, the Toeplitz operator induced by $\varphi$ is the operator $T_{\varphi}$ on $H^{2}$ defined by $T_{\varphi} f=P(\varphi f)$; here $P$ stands for the orthogonal projection in $L^{2}$ with range $H^{2}$. In this paper we shall study Weyl's theorem and its converse for the classes of all operators of the form $T_{f}^{-1} T_{g}$ and of the form $T_{g} T_{f}^{-1}$, where $f, g \in L^{\infty}$ and $T_{f}$ is invertible. In particular, we can prove the following results: (Theorem 1) $\sigma\left(T_{f}^{-1} T_{g}\right)$ is connected; (Theorem 3) Weyl's theorem holds for $T_{f}^{-1} T_{g}$; and (Theorem 4) if

Received by the editors August 6, 1971.

AMS 1970 subject classifications. Primary 47B35; Secondary 46J15.

Key words and phrases. Weyl's theorem, von Neumann's converse of Weyl's theorem, Toeplitz operators, Weyl spectrum.

${ }^{1}$ Research supported in part by the Sum:ner Research Initiation Award through the University Research Councii, University of Hawaii, 1971.

(c) American Mathematical Society 1972 
$f_{i}, g_{i} \in H^{\infty}+C$ where $C$ stands for the space of continuous complexvalued functions on the unit circle, and if $\omega\left(T_{f_{1}}^{-1} T_{g_{1}}\right)=\omega\left(T_{f_{2}}^{-1} T_{g_{2}}\right)$ is a proper subset of the unit circle, then $T_{f_{1}}^{-1} T_{g_{1}}$ and $T_{f_{2}}^{-1} T_{g_{2}}$ are essentially equivalent.

Recall that an operator $A$ is a Fredholm operator if it has a closed range and both a finite-dimensional kernel and cokernel. The class $F$ of Fredholm operators constitutes a multiplicative open semigroup in $L(H)$. For any $A \in F$, the index $i(A)$ is defined by the formula $i(A)=\operatorname{dim}[\operatorname{ker} A]-$ $\operatorname{dim}$ [coker $A$ ], and it is known that $i$ is a continuous integer-valued function and is invariant under compact perturbations.

Schechter [8] has observed that for any operator $A$,

$$
\omega(A)=\{\lambda \mid A-\lambda I \notin F\} \cup\{\lambda \mid A-\lambda I \in F \quad \text { and } \quad i(A-\lambda I) \neq 0\} .
$$

We need the following result of [4].

Lemma A. If $f \in L^{\infty}$, then $T_{f}$ is invertible if and only if $T_{f}$ is Fredholm of index zero.

THEOREM 1. $\sigma\left(T_{f}^{-1} T_{g}\right)$ is connected.

Proof. If $\lambda \notin \sigma\left(T_{f}^{-1} T_{g}\right), T_{f}^{-1} T_{g}-\lambda I$ is invertible and this implies that $T_{g-\lambda f}$ is invertible. Hence $\sigma\left(T_{f}^{-1} T_{g}\right)$ contains $R(g / f)$, the essential range of $\mathrm{g} / f$. We shall generalize a method in [11] to prove our theorem and hence we only give an outline of the proof. It suffices to show that if $\Gamma$ is any simple closed curve in the complex plane which is disjoint from $\sigma\left(T_{f}^{-1} T_{g}\right)$, then $\sigma\left(T_{f}^{-1} T_{g}\right)$ lies entirely inside or entirely outside $\Gamma$. Since $T_{g-i f}$ and $T_{(g-i f)^{-1}}$ are invertible for each $\lambda \in \Gamma$, the equations $T_{g-\lambda f} h=1$ and $T_{(g-\lambda f)^{-1}} k=1$ have solutions $h=h_{\lambda}$ and $k=k_{\lambda}$ in $H^{2}$ which can be shown $[11$, p. 579] to satisfy two differential equations whose solutions are

$$
h_{\lambda}=h_{\lambda_{0}} \exp \left(\int_{\lambda_{0}}^{\lambda} P((g / f)-\mu)^{-1} d \mu\right)
$$

and

$$
k_{\lambda}=k_{\lambda_{0}} \exp \left(-\int_{\lambda_{0}}^{\lambda} P((g / f)-\mu)^{-1} d \mu\right)
$$

respectively, where $\lambda_{0}$ is a fixed point of $\Gamma$. If one takes the path of integration to be the entire curve $\Gamma$, then it can be shown [11, p. 580] from (1) that $R(g / f)$ lies either entirely inside or entirely outside $\Gamma$. In the latter case, say, (1) and (2) show how to continue $h_{\lambda}$ and $k_{i}$ analytically to the inside of $\Gamma$. Now there is an explicit formula which gives the solution of the equation

$$
\left(T_{f}^{-1} T_{g}-\lambda I\right) \varphi=\psi
$$


in terms of $h_{\lambda}$ and $k_{\lambda}$ for $\lambda \notin \sigma\left(T_{f}^{-1} T_{g}\right)$, in fact

$$
\varphi=\varphi_{\lambda}=h_{\lambda} P\left(((g ; f)-\lambda)^{-1} k_{i} \psi\right)
$$

if $\psi \in H^{\infty}$. But then (4) shows us how to continue $\varphi=q_{i}$ analytically to the inside of $\Gamma$, and this continuation will provide the unique solution of (3) $\left[11\right.$, pp. 581-582]. Thus we have shown that $\sigma\left(T_{f}^{-1} T_{q}\right)$ lies entirely outside $\Gamma$. This completes the proof of the theorem.

Since $T_{f}^{-\mathrm{i}} T_{g}$ and $T_{g} T_{f}^{-1}$ are similar, we have the following corollary.

COROLlaRY 1.1. $\sigma\left(T_{g} T_{f}^{-1}\right)$ is connected.

If $f$ is in a special subclass of functions of $L^{\infty}$, we can have the foliowing theorem, in particular, we generailize some results in [6].

THEOREM 2. If $f=u h k$ where $h, k \in\left(H^{\infty}\right)^{-1}, u \in H^{\infty}+C$ such that $T_{u}$ is invertible, then $\sigma\left(T_{f}^{-1} T_{g}\right)=\sigma\left(T_{g / f}\right)=\sigma\left(T_{g} T_{f}^{-1}\right)$.

Proof. It suffices to prove $\sigma\left(T_{f}^{-1} T_{g}\right)=\sigma\left(T_{g / f}\right)$ only. Since

$$
T_{f}^{-1} T_{g}=\left(T_{u} T_{h}\right)^{-1}\left(T_{g / h k} T_{u}^{-1}\right)\left(T_{u} T_{h}\right)
$$

we have $\sigma\left(T_{f}^{-1} T_{g}\right)=\sigma\left(T_{g / h k} T_{u}^{-1}\right)$.

By Schechter's characterization of Weyl spectrum and Lemma A, we have $\sigma\left(T_{f}^{-1} T_{g}\right)=\omega\left(T_{f}^{-1} T_{\varepsilon}\right)[6]$. Since $u \in H^{\infty}+C$ and $T_{u}$ is invertible, we have $T_{u}^{-1}=T_{1 / u}+K$, where $K$ is a compact operator [10, Theorem 6] and $1 / u \in H^{\infty}+C[5]$; and since Weyl's theorem holds for any Toeplitz operator and $\sigma\left(T_{j}\right)=\omega\left(T_{f}\right)[4]$. we have

$$
\begin{aligned}
\sigma\left(T_{f}^{-1} T_{g}\right) & =\sigma\left(T_{g / h k} T_{u}^{-1}\right)=\omega\left(T_{g / h k} T_{u}^{-1}\right) \\
& =\omega\left(T_{g / f}+K\right)=\omega\left(T_{g / f}\right)=\sigma\left(T_{g / f}\right) .
\end{aligned}
$$

The proof is complete.

THEOREM 3. Weyl's theorem holds for $T_{i}^{-1} T_{g}$.

ProOF. If there were a $\lambda \in \sigma_{00}\left(T_{f}^{-1} T_{g}\right)$, this implies that $\{\lambda\}=\sigma\left(T_{f}^{-1} T_{g}\right)$ by Theorem 1. Since $R(g / f) \subset \sigma\left(T_{\dot{f}}^{-1} T_{g}\right)$ and it is never empty, $R(g \mid f)=\{\lambda\}$. It follows that $g=\lambda f$ a.e. and $T_{f}^{-1} T_{g}=\lambda_{c} I$ and $\lambda$ is an eigenvalue for $T_{f}^{-1} T_{0}$ of infinite multiplicity, which contradicts the fact $\lambda \in \sigma_{00}\left(T_{f}^{-1} T_{g}\right)$. Therefore $\sigma_{00}\left(T_{f}^{-1} T_{\vartheta}\right)=\varnothing$.

On the other hand, by Schechter's characterization of Weyl spectrum and Lemma $\mathrm{A}$, we have $\sigma\left(T_{f}^{-1} T_{g}\right)=\omega\left(T_{f}^{-1} T_{g}\right)$. Therefore Weyl's theorem holds for $T_{f}^{-1} T_{g}$. The proof is complete.

COROLlaRY 3.1. Weyl's theorem holds for $T_{a} T_{f}^{-1}$.

COROLlaRY 3.2. If $f=u h k$ where $h, k \in\left(H^{\infty}\right)^{-1}, u \in H^{\infty}+C$ such that $T_{u}$ is invertible, then $\left\|T_{f}^{-1} T_{g}+K\right\| \geqq\left\|T_{\text {gif }}\right\|$ for each compact operator $K$. 
Proof. By Theorems 2 and 3, we have

Therefore,

$$
\sigma\left(T_{g / f}\right)=\sigma\left(T_{f}^{-1} T_{g}\right)=\omega\left(T_{f}^{-1} T_{g}\right)
$$

$$
\left\|T_{g / f}\right\|=r\left(T_{g / f}\right)=r\left(T_{f}^{-1} T_{g}\right) \leqq r\left(T_{f}^{-1} T_{g}+K\right) \leqq\left\|T_{f}^{-1} T_{g}+K\right\|
$$

for each compact operator $K$, where $r(A)$ denotes the spectral radius of $A$. The proof is complete.

It is easily seen that $T_{f_{1}}^{-1} T_{g_{1}}$ and $T_{f_{2}}^{-1} T_{g_{2}}$ are not essentially equivalent in general, even if $\omega\left(T_{f_{1}}^{-1} T_{g_{1}}\right)=\omega\left(T_{f_{2}}^{-1} T_{g_{2}}\right)$; in fact we can take $f_{1}=f_{2}=1$, $g_{1}=z$ and $g_{2}=\bar{z}$.

THEOREM 4. If $f_{i}, g_{i} \in H^{\infty}+C$ such that $T_{f_{i}}$ is invertible for each $i=1,2$ and if $\omega\left(T_{f_{1}}^{-1} T_{g_{1}}\right)=\omega\left(T_{f_{2}}^{-1} T_{g_{2}}\right)$ is a proper subset of the unit circle, then $T_{f_{1}}^{-1} T_{g_{1}}$ and $T_{f_{2}}^{-1} T_{g_{2}}$ are essentially equivalent. Similarly for $T_{g_{i}} T_{f_{i}}^{-1}, i=1,2$.

Proof. If $f, g \in H^{\infty}+C$ and if $T_{f}$ is invertible, then $T_{f}^{-1}=T_{1 / f}+K$ and $T_{h} T_{g}=T_{h g}+J$, where $K$ and $J$ are compact operators [10]. It follows that $T_{f_{i}}^{-1} T_{g_{i}}=T_{g_{i} / f_{i}}+K_{i}, i=1,2$, where $K_{i}$ are compact operators and $\omega\left(T_{g_{1} / f_{1}}\right)=$ $\omega\left(T_{g_{2} / f_{2}}\right)=\sigma\left(T_{g_{i} / f_{i}}\right)=\omega\left(T_{f_{i}}^{-1} T_{g_{i}}\right)$. Therefore, $\sigma\left(T_{g_{i} / f_{i}}\right)$ is a proper subset of the unit circle for each $i=1,2$, and hence $T_{g_{i} / f_{i}}=U_{i}+J_{i}, i=1,2$, where $U_{i}$ are unitary operators and $J_{i}$ are compact operators [10]. It follows that $\omega\left(U_{1}\right)=\omega\left(U_{2}\right)=\sigma\left(T_{g_{i} / f_{i}}\right)$. Hence there is a unitary operator $U$ such that $U_{1}-U U_{2} U^{*}$ is compact [2]. It is easy to check that $T_{f_{1}}^{-1} T_{\sigma_{1}}-U\left(T_{f_{2}}^{-1} T_{\sigma_{2}}\right) U^{*}$ is compact for this $U$. Hence $T_{f_{1}}^{-1} T_{g_{1}}$ and $T_{f_{2}}^{-1} T_{g_{2}}$ are essentially equivalent. The proof is complete.

Corollary 4.1. If $g_{i} \in H^{\infty}+C, i=1,2$, and if $\omega\left(T_{g_{1}}\right)=\omega\left(T_{g_{2}}\right)$ is a proper subset of the unit circle, then $T_{g_{1}}$ and $T_{g_{2}}$ are essentially equivalent.

CoROllary 4.2. If $f_{i} \in H^{\infty}+C$ such that $T_{f_{i}}$ is invertible for each $i=1,2$ and $\omega\left(T_{f_{1}}^{-1}\right)=\omega\left(T_{f_{2}}^{-1}\right)$ is a proper subset of the unit circle, then $T_{f_{1}}^{-1}$ and $T_{f_{2}}^{-1}$ are essentially equivalent.

THEOREM 5. If $f_{i} \in H^{\infty}+C$ for each $i=1,2$, then $\operatorname{Re} \omega\left(T_{g_{1}} T_{f_{1}}^{-1}\right)=$ $\operatorname{Re} \omega\left(T_{g_{2}} T_{f_{2}}^{-1}\right)$ for some $g_{i} \in L^{\infty}, i=1,2$, if and only if $\operatorname{Re}\left(T_{g_{1}} T_{f_{1}}^{-1}\right)$ and $\operatorname{Re}\left(T_{g_{2}} T_{f_{2}}^{-1}\right)$ are essentially equivalent, where $\operatorname{Re}\left(T_{g_{i}} T_{f_{i}}^{-1}\right)$ denotes the real part of $T_{g_{i}} T_{f_{i}}$ for each $i=1$, 2. Similarly for $\operatorname{Im}\left(T_{g_{i}} T_{f_{i}}^{-1}\right)$.

Proof. It suffices to prove the real part only. Since $f_{i} \in H^{\infty}+C$ for $i=1$, 2 we have

$$
\omega\left(T_{g_{i}} T_{f_{i}}^{-1}\right)=\omega\left(T_{g_{i} / f_{i}}\right)=\sigma\left(T_{g_{i} / f_{i}}\right) .
$$

It is known that $\operatorname{Re} \sigma\left(T_{h}\right)=\sigma\left(\operatorname{Re} T_{h}\right)$ for any Toeplitz operator [3]. Therefore, $\omega\left(\operatorname{Re} T_{g_{1} / f_{1}}\right)=\omega\left(\operatorname{Re} T_{g_{2} / f_{2}}\right)$ if $\operatorname{Re} \omega\left(T_{g_{1}} T_{f_{1}}^{-1}\right)=\operatorname{Re} \omega\left(T_{g_{2}} T_{f_{2}}^{-1}\right)$ and 
hence $\operatorname{Re}\left(T_{g_{1} / f_{1}}\right)$ and $\operatorname{Re}\left(T_{g_{2} / f_{2}}\right)$ are essentially equivalent by von Neumann's theorem [2, Theorem 8.1]. Hence $\operatorname{Re}\left(T_{g_{1}} T_{f_{1}}^{-1}\right)$ and $\operatorname{Re}\left(T_{g_{2}} T_{f_{2}}^{-1}\right)$ are essentially equivalent by the relations $T_{g_{i}} T_{f_{i}}^{-1}=T_{g_{i} / f_{t}}+K_{i}$, where $K_{i}$ are compact operators for $i=1,2$.

Conversely, if $\operatorname{Re}\left(T_{g_{1}} T_{f_{2}}^{-1}\right)$ and $\operatorname{Re}\left(T_{g_{2}} T_{f_{2}}^{-1}\right)$ are essentially equivalent, then $\omega\left(\operatorname{Re} T_{s_{1}} T_{f_{1}}^{-1}\right)=\omega\left(\operatorname{Re} T_{g_{2}} T_{f_{2}}^{-1}\right)$. Therefore, we have

$$
\begin{aligned}
\operatorname{Re} \omega\left(T_{g_{i}} T_{f_{i}}^{-1}\right) & =\operatorname{Re} \omega\left(T_{g_{i} / f_{i}}\right)=\operatorname{Re} \sigma\left(T_{g_{j} / f_{i}}\right) \\
& =\sigma\left(\operatorname{Re} T_{g_{i} / f_{i}}\right)=\omega\left(\operatorname{Re} T_{g_{i} / f_{i}}\right)=\omega\left(\operatorname{Re} T_{g_{i}} T_{f_{i}}^{-1}\right),
\end{aligned}
$$

and hence, $\operatorname{Re} \omega\left(T_{g_{1}} T_{f_{1}}^{-1}\right)=\operatorname{Re} \omega\left(T_{g_{2}} T_{f_{2}}^{-1}\right)$. The proof is complete.

COROllary 5.1. $\operatorname{Re} \omega\left(T_{g_{1}}\right)=\operatorname{Re} \omega\left(T_{g_{2}}\right)$ if and only if $\operatorname{Re}\left(T_{g_{1}}\right)$ and $\operatorname{Re}\left(T_{g_{2}}\right)$ are essentially equivalent.

\section{REFERENCES}

1. S. K. Berberian, An extension of Weyl's theorem to a class of not necessarily normal operators, Michigan Math. J. 16 (1969), 273-279. MR 40 \#3335.

2. - The Weyl spectrum of an operator, J. Math. Mech. 20 (1970), 529-544.

3. Conditions on an operator implying $\operatorname{Re} \sigma(T)=\sigma(\operatorname{Re} T)$, Trans. Amer. Math. Soc. 154 (1971), 267-273.

4. L. A. Coburn, Weyl's theorem for nonnormal operators, Michigan Math. J. 13 (1966), 285-288. MR 34 \#1846.

5. R. G. Douglas, Toeplitz and Wiener-Hopf operators in $H^{\infty}+C$, Bull. Amer. Math. Soć. 74 (1968), 895-899. MR 37 \#4648.

6. E. M. Klein, Toeplitz operators and Weyl spectra, Thesis, Northwestern University, Evanston, Ill., 1971.

7. J. von Neumann, Charakterisierung des Spektrums eines Integraloperators, Hermann, Paris, 1935.

8. M. Schechter, Invariance of the essential spectrum, Bull. Amer. Math. Soc. 71 (1965), 365-367. MR 31 \#3710.

9. W. Sikonia, The von Neumann converse of Weyl's theorem (preprint).

10. J. G. Stamptil, On hyponormal and Toeplitz operators, Math. Ann. 183 (1969), 328-336. MR 40 \#4798.

11. H. Widom, Tocplitz operators on $H_{p}$, Pacific J. Math. 19 (1966), 573-582. MR 34 \#1859.

Department of Mathematics, University of Hawail, Honolulu, Hawail 96822 